\title{
Infiltración con bupivacaína de la incisión de Pfannenstiel para la analgesia pos cesárea
}

\author{
Alfi Contreras Benítez ${ }^{1,2}$. José Ramón Urdaneta Machado, $P h D^{3}$. Nasser Baabel \\ Zambrano, $P h D .^{2,4}$ \\ ${ }^{1}$ Anestesiólogo. Cátedra de Anatomía Humana. Facultad de Odontología. Universidad del Zulia. ${ }^{2}$ Instituto Venezolano de \\ los Seguros Sociales. Hospital "Dr. Adolfo Pons" ${ }^{3}$ Cátedra de Anatomía. Departamento de Morfofisiopatología. Facultad \\ de Medicina. Universidad del Zulia. ${ }^{4}$ Cátedra de Práctica Profesional de Ginecología y Obstetricia. Facultad de Medicina. \\ Universidad del Zulia. Venezuela.
}

\section{RESUMEN}

Objetivo: Comparar la efectividad analgésica de la infiltración con Bupivacaína de la incisión de Pfannenstiel respecto a un placebo para la analgesia posoperatoria de gestantes llevadas a cesárea segmentaria en el Hospital "Dr. Adolfo Pons" de Maracaibo, estado Zulia, Venezuela. Métodos: Investigación comparativa y aplicada, con diseño cuasi-experimental, de casos y controles, contemporáneo y de campo, donde se incluyeron 60 gestantes planificadas para cesárea segmentaria electiva, divididas aleatoriamente en dos grupos pareados para infiltrarles la incisión de Pfannenstiel con $30 \mathrm{~mL}$ de Bupivacaína al $0,25 \%$ (75 mg) o solución salina. La intensidad del dolor se evaluó mediante la escala visual análoga (EVA). Resultados: En cuanto a la intensidad del dolor, tanto en reposo como en movimiento, se encontraron diferencias altamente significativas a favor del grupo que recibió la infiltración incisional con Bupivacaína durante las primeras seis horas del postoperatorio $(p<0,001)$; posterior a las 8 horas del posoperatorio no hubo diferencias significativas entre los dos grupos evaluados. De igual manera, estas pacientes presentaron un mayor tiempo libre de dolor, que sobrepasaba las 4 horas $(251 \pm 14$ minutos vs. $220 \pm 11$ minutos; $\mathrm{p}<0,001)$, menores requerimientos analgésicos $(199,37 \pm 0,15 \mathrm{mg}$ vs. $298,04 \pm 1,96 \mathrm{mg} ; \mathrm{p}<0,001)$ y con menos efectos adversos $(p<0,05)$. Conclusión: La infiltración de la incisión de Pfannenstiel es efectiva para la analgesia postoperatoria de la cesárea segmentaria, prolongado el tiempo libre de dolor y disminuyendo los requerimientos de analgésicos.

\section{PALABRAS CLAVE: Analgesia posoperatoria, bupivacaína, cesárea, Pfannenstiel, infiltración anestésica}

\section{SUMMARY}

Aim: To compare the analgesic effectiveness of Pfannenstiel incision infiltration with bupivacaine over a placebo for post-operative analgesia in cesarean section in pregnants attending at the Hospital "Dr. Adolfo Pons" in Maracaibo, Zulia state, Venezuela. Methods: A comparative and applied research, with quasiexperimental, case-control, contemporary and field design, which included 60 pregnant women scheduled for elective cesarean section, divided randomly into two groups matched for Pfannestiel incision infiltration with $30 \mathrm{~mL}$ Bupivacaine $0.25 \%$ (75 mg) or saline solution. Pain intensity was assessed by visual analog scale (VAS). Results: In terms of pain intensity at rest and in motion, were found highly significant differences in favor of the group receiving the incisional infiltration with bupivacaine during the first six hours after surgery $(p<0.001)$; not later than 8 hours after surgery where in all measurements, were found no significant differences between the two tested groups. Similarly, these patients had higher pain free time, surpassing the 4 hours ( $251 \pm 14$ minutes vs. $220 \pm 11$ minutes; $p<0.001)$, lower analgesic requirements $(199.37 \pm 0.15$ 
mg vs. $298.04 \pm 1.96 \mathrm{mg} ; \mathrm{p}<0.001)$ and fewer adverse effects $(p<0.05)$. Conclusion: The infiltration of the Pfannenstiel incision is effective for postoperative analgesia in cesarean section, prolonged pain-free time and decreasing analgesic requirements.

\section{KEY WORDS: Post operatory analgesia, bupivacaine, caesarea, Pfannenstiel, anaesthetic infiltration}

\section{INTRODUCCIÓN}

La cesárea en los últimos 30 años viene exhibiendo un aumento continuo en su tendencia, presentando tasas con amplia variación entre los países del mundo, las cuales oscilan entre 0,4 a $40 \%$ (1-3). Han contribuido al aumento de la tasa de cesárea primaria, la mayor laxitud en su indicación y una creciente seguridad del parto por cesárea (4). En los EEUU se realizan cada año más de un millón de cesáreas, representando más del $30 \%$ de los nacimientos (5); la tasa de cesárea aumentó de $5 \%$ en 1970 a 32,9\% en 2009 (6), proyectándose para 2020 un 56,2\% $(4,7)$.

Lo mismo sucede en otros países del continente, como Argentina donde se ha registrado una frecuencia global de $30 \%$ y superior al $50 \%$ en centros privados (3). En Venezuela se reportó una tendencia al ascenso con un marcado incremento, desde 4,6/100 nacimientos entre 1970-1972 a 43,2/100 nacimientos en 2010 (8); siendo su principal indicación la desproporción fetopélvica (2).

Comparada con el parto vaginal, la cesárea está asociada a una mayor necesidad de la utilización de analgésicos debido al dolor posoperatorio (9); la cesárea generalmente induce dolor moderado a severo que dura generalmente unas 48 horas y es la principal preocupación de las pacientes (5,10-12). El dolor puede resultar en serias morbilidades si no es manejado, aunque existe una gran cantidad de variación en la tolerancia al dolor y por tanto de la necesidad de analgésicos (13).

Está establecido que el dolor posoperatorio puede ocasionar incomodidad en la paciente, prolonga la recuperación e incrementa los costos hospitalarios, y en los casos de cesárea debe agregársele la interferencia que pueda tener con la deambulación, la lactancia y el apego materno temprano con el neonato (14).

Comúnmente se han utilizado los opioides para el alivio del dolor posoperatorio después de esta intervención, por administración intratecal antes de la sección o parenteral después de la operación (11). Se ha reportado que el consumo promedio de morfina en las primeras 24 horas posteriores a la cesárea es de alrededor 35-54,5 mg (15), sin embargo, para obtener una analgesia de buena calidad y de larga duración durante el postoperatorio, deben utilizarse dosis altas de opioides, pero el riesgo de complicaciones como depresión respiratoria, retención urinaria, prurito, náuseas y vómitos puede impedir la comodidad del paciente (16). Mientras otras técnicas de analgesia postoperatoria, como la morfina epidural o anestésicos locales también tienen límites, pues requieren una vigilancia clínica prolongada (11).

La infiltración incisional con anestésicos locales es usada en gran parte de los regímenes de analgesia multimodal; consiste en la inyección de una solución de anestésicos locales directamente en el tejido a anestesiar (dermis o tejido subcutáneo), por lo general lidocaína, procaína, mepivacaína o bupivacaína (17). La analgesia del tejido subcutáneo puede aumentar la eficacia de la analgesia postoperatoria (18); constituyendo una forma sencilla para aliviar el dolor (19). Tiene una duración de acción limitada, menor de 5 horas, contribuyendo a la disminución de la demanda de analgésicos sistémicos, sin aumento de la dehiscencia de la herida o infección (11).

En el caso de la cesárea segmentaria, el dolor posoperatorio suele ser de intensidad moderada a severa, con dos componentes: un componente somático, en relación con la cicatriz en la pared abdominal y un componente visceral, en relación con las contracciones uterinas $(12,18)$. Ésta intervención se efectúa por lo general mediante la incisión de Pfannenstiel, la cual se realiza a nivel de los dermatomos L1-L2, cuya inervación sensorial se realiza por los nervios ilioinguinal y iliohipogástrico (20). La infiltración de la incisión con anestésicos locales es una técnica que se considera parte integral del protocolo analgésico en pacientes programados para una cesárea, pues es simple, fácil de realizar y tiene como objetivo dar un alivio óptimo del dolor con efectos secundarios mínimos, disminuyendo la demanda de analgesia de rescate y sin interferir en la relación madre-hijo; favoreciendo la lactancia materna, su bienestar psicológico, la movilidad, la actividad intestinal y así la ingesta de alimentos más temprana, con una rápida rehabilitación postoperatoria (11).

Diversos estudios clínicos han avalado la eficacia de la infiltración local de la Bupivacaína en un amplio espectro de operaciones, incluyendo la cesárea segmentaria $(18,21)$, histerectomía abdominal $(22,23)$, artroplastia (24), cirugía de columna lumbar (25), prostactectomía retropúbica (26), cirugía de varicocele $(27)$, hernioplastia $(28,29)$, cirugía de corrección paladar hendido en niños (30), amigdalectomía (31), cirugías ambulatorias (32), entre otras. Sin embargo, los resultados han 
sido controversiales, desde ser beneficiosos a no presentar ninguna utilidad $(18,33)$.

El propósito de este estudio es determinar la efectividad de la infiltración con Bupivacaína de la incisión de Pfannenstiel post cesárea.

\section{MÉTODOS}

Investigación de tipo comparativa con diseño cuasi experimental de casos y controles, realizada en embarazadas ingresadas a la unidad de partos del Hospital "Dr. Adolfo Pons" de Maracaibo, estado Zulia, Venezuela. Se seleccionó una muestra intencionada a simple ciego conformada por 60 embarazadas mediante la aplicación de un muestreo aleatorio sistematizado. Para ello, la primera paciente seleccionada se le otorgó el número "1" asignada al Grupo "A" (Casos) para recibir una instilación de Bupivacaína en la incisión, mientras que la siguiente paciente se tomó como la número dos "2" y asignada al grupo "B" (Controles) para infiltración de solución salina. Así, todas las pacientes con números impares fueron los casos y las con números pares los controles.

Para la selección de los casos y controles, debieron cumplir con los siguientes criterios de inclusión: embarazadas mayores de 19 años y menores de 35 años, cesárea electiva bajo anestesia peridural, ASA I (pacientes sanos) o ASA II (pacientes con enfermedad sistémica leve). Se excluyeron las pacientes con cesáreas de emergencia, embarazo gemelar, con anestesia general o subaracnoidea, contraindicación o hipersensibilidad a los opiodes o a los antiinflamatorios no esteroideos (AINES), trastornos hipertensivos del embarazo, enfermedades sistémicas, crónicas y/o degenerativas o psiquiátricas, IMC>30 Kg/m2), esterilización quirúrgica pos operación cesárea, y con historia de dolor crónico y/o uso crónico de analgésicos o con adicciones a drogas y/o psicotrópicos.

A cada paciente se le solicitó el consentimiento informado por escrito, previa explicación del procedimiento a seguir, para su inclusión en el protocolo de estudio; el cual fue previamente aprobado por el comité de bioética de la institución ámbito de la investigación; seguidamente, se procedió a realizar la consulta médica pre-anestésica y mediante el interrogatorio clínico se verificaron datos clínicos y epidemiológicos. Las cesáreas fueron programadas para el día siguiente, debiendo estar todas las pacientes en ayuno de 8 horas y recibieron previo al inicio de la cirugía infusión endovenosa de solución Ringer Lactato (500 mL) en 15 minutos; además en aquellos casos donde no se haya cumplido con el ayuno se les administró $50 \mathrm{mg}$ de ranitidina en bolo endovenoso directo y $10 \mathrm{mg}$ de metoclopramida en infusión, 15 minutos antes de la aplicación de la anestesia.

La técnica anestésica utilizada fue la peridural con punción entre L3 y L4 o entre L4 y L5 en posición sentada con la columna en máxima flexión, previa infiltración local con lidocaína al $2 \%$. Se usó aguja de Tuohy calibre 18. Se administró una mezcla anestésica de lidocaína al $2 \%$ de $400 \mathrm{mg}(10 \mathrm{~mL})$, fentanilo $100 \mathrm{mcg}(2 \mathrm{~mL})$ y clonidina $75 \mathrm{mcg}(0,5$ $\mathrm{mL}$ ). El bloqueo sensorial más profundo fue verificado y confirmado en el nivel de T3 a T5; el bloqueo motor se midió con la escala de Bromage modificada ( 0 , no bloqueo; 1 , incapacidad de elevar la pierna extendida; 2 , incapacidad de flexionar la rodilla; y 3 , incapacidad de flexionar el tobillo y el pie).

La intervención quirúrgica comenzó con bloqueo sensorial en el dermatoma T6, todas las cesáreas se realizaron mediante incisión de Pfannenstiel, con apertura peritoneal. La infiltración de la incisión la realizó el cirujano, quien instiló directamente sobre los planos anatómicos de la incisión antes de cierre de la piel $30 \mathrm{~mL}$ de Bupivacaína al $0,25 \%$ (75mg) o $30 \mathrm{~mL}$ de solución salina en el grupo control; $10 \mathrm{~mL}$ para la aponeurosis y $10 \mathrm{ml}$ por vía subcutánea en cada uno de los bordes superior e inferior de la incisión, usando tres jeringas de $10 \mathrm{~mL}$ con aguja subcutánea 23G. Esta infiltración en tres partes bien diferenciadas se eligió para estandarizar la práctica y reducir las diferencias relacionadas con el operador. Asimismo, recibieron una dosis endovenosa de Ketoprofeno (100 mg) y Metoclorpropamida (10 mg) o Ondansetrón (4 mg); además de 20 UI de Oxitócina titulada en $500 \mathrm{~mL}$ de solución salina al 0,9\%.

Para la evaluación de la analgesia posoperatoria, se midió el dolor durante las primeras 24 horas por medio del uso de una escala visual análoga (EVA) con valores desde 0 (sin dolor) a 10 (peor dolor imaginable), señalando en una regla de 10 centímetros el punto que según la paciente represente el dolor que manifiesta (Figura 1). Se evaluó el dolor estático (en reposo) y dinámico (al toser, sentarse o movilizar la parte afectada) cada 2 horas durante las primeras 8 horas y luego cada 8 horas. En ambos grupos cualquier analgesia posoperatoria fue administrada en la sala de recuperación vigilando el dolor y cuando la paciente lo solicite o si la evaluación del dolor fue $\geq 4$, se administró una dosis endovenosa de $100 \mathrm{mg}$ de Ketoprofeno cada 8 horas.

Para la valoración de la eficacia de la infiltración con Bupivacaína o solución salina en la incisión de Phannenstiel, se midió la necesidad de analgesia de rescate, valorada como el requerimiento adicional de analgésicos mediante el consumo de los mismos expresados en miligramos y el tiempo libre de dolor, datos que se obtendrán mediante el interrogatorio de la paciente y verificación en la historia clínica y la tarjeta enfermería. Asimismo, se comprobaron la presencia o no de efectos indeseados como la presencia de hematomas, enrojecimiento, dolor, calor o secreciones en la herida quirúrgica. 


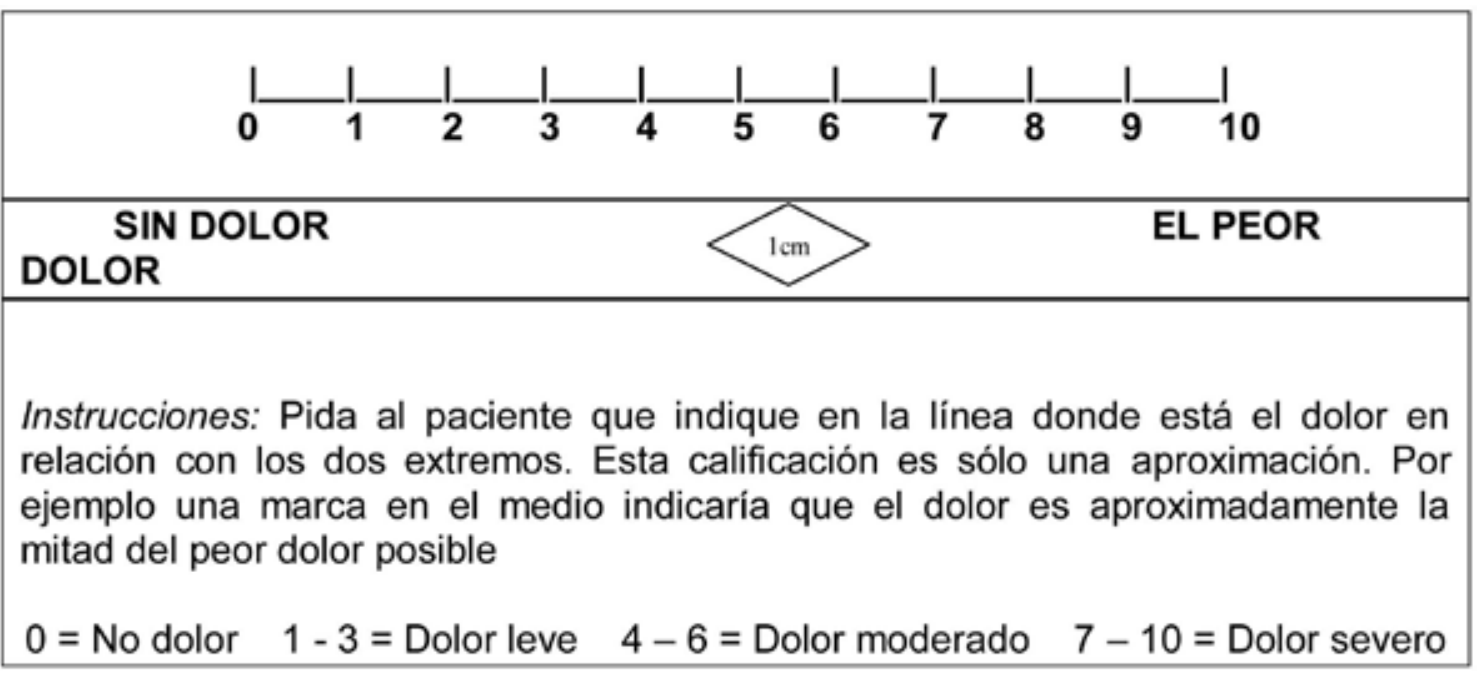

Figura 1. Escala visual análoga (EVA).

Los datos obtenidos se analizaron y fueron expresados mediante frecuencias absolutas o relativas (\%), medidas de tendencia central (media) y de dispersión ( $\pm \mathrm{DE})$. Para efectuar las comparaciones entre los grupos se utilizó el test de Student para los datos cuantitativos y la prueba del Chi-cuadrado para los cualitativos; ambos estadísticos con un nivel de confianza del $95 \%(p<0,05)$.

\section{RESULTADOS}

En la Tabla I se pueden apreciar algunas características de la muestra evaluada; evidenciándose que la edad fue en promedio de 24 años en ambos grupos. Asimismo, la edad gestacional por el diseño del estudio se encontró en el tercer trimestre de la gestación, alrededor de las 39 semanas para el grupo $A$ y de las 40 semanas para el grupo B $(p<0,001)$; para ambos grupos los promedios de la menarquía se ubicaron a los 12 años y los de la paridad y número de parejas en 2 . El análisis estadístico efectuado solo determinó diferencias altamente significativas en cuanto a la edad gestacional, el resto de las características estudiadas no mostró diferencias, lo cual comprueba la homogeneidad de los grupos.

Tabla I

CARACTERIZACIÓN DE LA MUESTRA: ANÁLISIS CUANTITATIVO

\begin{tabular}{lccc}
\hline Análisis descriptivo & $\begin{array}{c}\text { Grupo } A^{*} \\
\text { (Casos) } \\
\text { Media } \pm \text { DE }\end{array}$ & $\begin{array}{c}\text { Grupo B* } \\
\text { (Controles) } \\
\text { Media } \pm \text { DE }\end{array}$ & $\begin{array}{c}\text { Valor } \\
\mathrm{p}^{\star *}\end{array}$ \\
\hline Edad & $24,07 \pm 1,05$ & $24,73 \pm 2,95$ & 0,253 \\
Edad gestacional & $39,15 \pm 0,59$ & $39,60 \pm 0,43$ & 0,001 \\
Menarquia & $11,97 \pm 0,72$ & $11,83 \pm 1,12$ & 0,584 \\
Sexarquia & $17,83 \pm 1,37$ & $17,23 \pm 1,72$ & 0,616 \\
Número de parejas & $2,07 \pm 1,14$ & $1,93 \pm 1,01$ & 0,634 \\
Paridad & $1,95 \pm 0,86$ & $2,23 \pm 1,15$ & 0,290 \\
Talla $(\mathrm{cm})$ & $166,33 \pm 5,89$ & $168,50 \pm 8,19$ & 0,276 \\
Peso $(\mathrm{Kg})$ & $74,20 \pm 7,70$ & $76,80 \pm 8,85$ & 0,192
\end{tabular}

DE: Desviación estándar. ${ }^{\star} n=30$ para cada grupo. ${ }^{\star \star}$ Prueba T de Student. $p<0,05$ valor significativo. 
La Tabla II muestra el análisis cualitativo de las características de la muestra estudiada, donde se evidencia en ambos grupos una frecuencia elevada de mujeres en soltería o unión libre, bajos niveles educativos, procedente del medio urbano, viviendas precarias o de interés social y un bajo ingreso mensual. En ambos grupos prevalecieron la clase ASA II y la cesárea anterior.

Tabla II

CARACTERIZACIÓN DE LA MUESTRA: ANÁLISIS CUALITATIVO

\begin{tabular}{|c|c|c|c|c|}
\hline \multirow[t]{2}{*}{ Categorías } & \multicolumn{2}{|c|}{$\begin{array}{c}\text { Grupo A* } \\
\text { (Casos) }\end{array}$} & \multicolumn{2}{|c|}{$\begin{array}{c}\text { Grupo B* } \\
\text { (Controles) }\end{array}$} \\
\hline & $\mathrm{Fr}$ & $\%$ & $\mathrm{Fr}$ & $\%$ \\
\hline $\begin{array}{l}\text { Estado civil: } \\
\text { Solteras }\end{array}$ & 6 & 20,00 & 10 & 33,33 \\
\hline Concubinas & 21 & 70,00 & 17 & 56,67 \\
\hline Casadas & 3 & 10,00 & 3 & 10,00 \\
\hline \multicolumn{5}{|l|}{ Procedencia: } \\
\hline Rural & 2 & 6,67 & 3 & 10,00 \\
\hline Urbana & 28 & 93,33 & 27 & 90,00 \\
\hline \multicolumn{5}{|l|}{ Grado de instrucción: } \\
\hline Analfabetas & 3 & 10,00 & 2 & 6,66 \\
\hline Primaria incompleta & 3 & 10,00 & 2 & 6,66 \\
\hline Primaria completa & 5 & 16,67 & 3 & 10,00 \\
\hline Secundaria incompleta & 12 & 40,00 & 5 & 16,67 \\
\hline Secundaria completa & 5 & 16,67 & 9 & 30,00 \\
\hline Técnica/universitaria & 2 & 6,66 & 9 & 30,00 \\
\hline \multicolumn{5}{|l|}{ Tipo de vivienda: } \\
\hline Rancho & 13 & 43,33 & 8 & 26,67 \\
\hline Vivienda de interés social & 7 & 23,33 & 14 & 46,66 \\
\hline Casa u Apartamento & 10 & 33,33 & 8 & 26,67 \\
\hline \multicolumn{5}{|l|}{ Ingreso mensual: } \\
\hline Menos del sueldo mínimo & 16 & 53,33 & 11 & 36,67 \\
\hline Sueldo mínimo & 4 & 13,33 & 10 & 33,33 \\
\hline Mas del sueldo mínimo & 10 & 33,33 & 9 & 10,00 \\
\hline \multicolumn{5}{|l|}{ Control prenatal: } \\
\hline Mal controlado & 17 & 56,67 & 19 & 43,33 \\
\hline Bien controlado & 13 & 43,33 & 11 & 36,67 \\
\hline \multicolumn{5}{|l|}{ Riesgo anestésico: } \\
\hline ASA I & 12 & 40,00 & 14 & 46,67 \\
\hline ASA II & 18 & 60,00 & 16 & 53,33 \\
\hline \multicolumn{5}{|l|}{ Indicación de cesárea: } \\
\hline Cesárea anterior & 21 & 70,00 & 22 & 73,33 \\
\hline Desproporción feto-pélvica & 7 & 23,33 & 5 & 16,67 \\
\hline Presentación viciosa & 2 & 6,67 & 3 & 10,00 \\
\hline
\end{tabular}

${ }^{*} \mathrm{n}=30$ para cada grupo 
En la Tabla III se presenta la efectividad analgésica de la infiltración con Bupivacaína en la incisión de Pfannenstiel para la analgesia posoperatoria. En cuanto a la intensidad del dolor, tanto en reposo como en movimiento se encontraron diferencias significativas a favor del grupo que recibió la infiltración incisional con Bupivacaína durante las primeras seis horas del postoperatorio $(p<0,001)$; no así posterior a las 8 horas del postoperatorio donde en todas las mediciones realizadas no se encontraron diferencias significativas entre los dos grupos evaluados. En las pacientes que se infiltró Bupivacaína presentaron un mayor tiempo libre de dolor, que sobrepasaba las 4 horas, comparadas con la infiltración salina $(p=0,000)$; asimismo, en estas pacientes los requerimientos analgésicos fueron menores $(p=0,000)$.

En cuanto a los efectos adversos (Tabla IV), se encontró una menor prevalencia en el grupo que recibió la infiltración de la bupivacaína que en las que recibieron el placebo $(13,33 \%$ vs $20 \% ; p<0,05)$. Los efectos indeseables encontrados fueron menores, sin evidencias de alteraciones hemodinámicas ni de neurotoxicidad en ambos grupos. En el grupo A se reportaron dos pacientes con náuseas y vómitos postoperatorios, una con fiebre y una con seroma de la herida quirúrgica; mientras que en el grupo $B$ se detectaron 3 pacientes con náuseas y vómitos posoperatorios, dos con seroma y una infección de la herida quirúrgica.

\section{Tabla III}

EFECTIVIDAD ANALGÉSICA DE LA INFILTRACIÓN INCISIONAL DE BUPIVACAÍNA

\begin{tabular}{rccc}
\hline Análisis descriptivo & $\begin{array}{c}\text { Grupo } A^{*} \\
\text { (Casos) } \\
\text { Media } \pm \text { DE }\end{array}$ & $\begin{array}{c}\text { Grupo } B^{\star} \\
\text { (Controles) } \\
\text { Media } \pm \text { DE }\end{array}$ & $\begin{array}{c}\text { Valor } \\
p^{\star \star}\end{array}$ \\
\hline $\begin{array}{r}\text { Intensidad del dolor: } \\
\text { (EVA estático/cm) }\end{array}$ & & \\
2 HPO & & $2,9 \pm 0,9$ & 0,000 \\
4 HPO & $1,8 \pm 0,5$ & $4,5 \pm 1,1$ & 0,000 \\
6 HPO & $3,4 \pm 1,3$ & $4,4 \pm 0,8$ & 0,000 \\
8 HPO & $3,8 \pm 0,3$ & $3,9 \pm 1,3$ & 0,506 \\
16 HPO & $3,7 \pm 1,0$ & $3,5 \pm 0,7$ & 0,526 \\
24 HPO & $3,4 \pm 0,5$ & $3,3 \pm 0,8$ & 0,650
\end{tabular}

Intensidad del dolor:

(EVA dinámico/cm)

$\begin{array}{lrrr}2 \text { HPO } & 2,5 \pm 0,8 & 3,2 \pm 0,7 & 0,000 \\ 4 \text { HPO } & 4,3 \pm 0,6 & 5,4 \pm 0,9 & 0,000 \\ 6 \text { HPO } & 4,2 \pm 1,1 & 5,1 \pm 0,6 & 0,000 \\ 8 \text { HPO } & 4,0 \pm 0,9 & 4,2 \pm 0,8 & 0,366 \\ 16 \text { HPO } & 3,9 \pm 0,9 & 4,1 \pm 0,7 & 0,340 \\ 24 \text { HPO } & 3,6 \pm 0,5 & 3,7 \pm 0,7 & 0,526 \\ \text { de dolor (minutos) } & 251 \pm 14 & 220 \pm 11 & 0,000 \\ \text { os de analgésicos }(\mathrm{mg}) & 199,37 \pm 0,15 & 298,04 \pm 1,96 & 0,000\end{array}$

DE: Desviación estándar. HPO: Horas de posoperatorio. * $n=30$ para cada grupo. ** Prueba T de Student. $p<0,05$ valor significativo. 


\section{Tabla IV \\ EFECTOS ADVERSOS DE LA INFILTRACIÓN INCISIONAL DE BUPIVACAÍNA}

\begin{tabular}{|c|c|c|c|c|c|}
\hline \multirow[t]{2}{*}{ Análisis descriptivo } & \multicolumn{2}{|c|}{$\begin{array}{c}\text { Grupo A* } \\
\text { (Casos) }\end{array}$} & \multicolumn{2}{|c|}{$\begin{array}{l}\text { Grupo B* } \\
\text { (Controles) }\end{array}$} & \multirow[t]{2}{*}{ Valor $p^{\star \star}$} \\
\hline & $\mathrm{Fr}$ & $\%$ & $\mathrm{Fr}$ & $\%$ & \\
\hline Sin efectos adversos & 26 & 86,67 & 24 & 80,00 & 0,041 \\
\hline Con efectos adversos & 4 & 13,33 & 6 & 20,00 & \\
\hline
\end{tabular}

${ }^{*} \mathrm{n}=30 .{ }^{*}$ Prueba de Chi cuadrado. $\mathrm{p}<0,05$ significativo.

\section{DISCUSIÓN}

En esta serie, al igual que lo publicado en otras investigaciones nacionales, la cesárea anterior y la desproporción fetopélvica fueron las principales indicaciones para la realización de este procedimiento (2). Por tal motivo, es menester del profesional de la anestesiología garantizar un manejo adecuado del dolor postoperatorio en este número cada vez mayor de mujeres que experimentaran una intervención cesárea; la cual provoca dolor de moderado a severo en las primeras 48 horas que puede inducir a la administración de fármacos analgésicos como los opioides que ameritan vigilancia médica y pueden ocasionar efectos colaterales como depresión respiratoria, retención urinaria, prurito, así como nauseas y vómitos (11).

Al igual que en otros estudios, se encontró que los valores de la escala visual análoga fueron menores en los grupos donde se infiltro la herida quirúrgica con Bupivacaína (18). Contrario a los resultados presentados, en otra investigación donde se encontró que la infiltración continua a nivel subfascial con bupivacaína consigue disminuir el nivel de EVA tanto en reposo como en movimiento, especialmente a partir de las 16 horas siguientes a la cirugía (34); lo cual difiere con los resultados de esta serie donde la disminución de la intensidad del dolor fue menor en las primeras horas del postoperatorio.

De igual manera, similar a lo reportado en otras investigaciones, el requerimiento de analgésicos fue inferior en el grupo que recibió el anestésico local en comparación con el grupo que recibió un placebo. Al respecto, en el estudio efectuado por Demiraran y cols (18), se reportó un consumo de $483,3 \mathrm{mg}$ de tramadol en 24 horas en el grupo donde se infiltró la bupivacaína en comparación a 557,5 mg en el grupo que recibió un placebo $(p=0,007)$. Asimismo, Jabalameli y cols (35), encontraron que el número de pacientes que necesitaron morfina fueron significativamente diferentes entre las puérperas que se les infiltró solución salina en la herida operatoria que en las que se administró bupivacaína (105 mg vs $87 \mathrm{mg} ; \mathrm{p<0,05);} \mathrm{mientras} \mathrm{que} \mathrm{otro}$ autores reportaron que la infiltración de bupivacaína disminuía el consumo de analgésicos vía oral o intravenosa, sin efectos adversos y con una elevada satisfacción de los pacientes $(34,36)$.

También se ha reportado el uso de otros anestésicos locales, como Ropivacaína, en la infiltración de la herida operatoria en la cesárea segmentaria; encontrándose diferencias significativas en la evaluación del dolor con EVA al compararlo con solución salina en las primeras 4 horas, más no así en las otras mediciones realizadas durante el puerperio inmediato (37); hallazgo similar al encontrado en esta serie de casos donde se encontraron diferencias altamente significativas sólo durante las primeras horas del posoperatorio inmediato.

Concordante al estudio efectuado, Nguyen y cols (11), utilizaron Ropivacaína en la infiltración de la incisión de Pfannenstiel; hubo una reducción del $30 \%$ en el consumo total de analgésicos, especialmente los opiáceos en el primeras 24 horas, pero también aumenta significativamente el intervalo de tiempo hasta la primera solicitud de un analgésico (4:20 $\pm 2: 26$ horas vs. $2: 42 \pm 1: 30$ horas), con valores de $p<0,0001$ para paracetamol, ketoprofeno y nalbufina. En tanto que otros estudios determinaron que la infiltración de Ropivacaína disminuía tanto el dolor posoperatorio como la necesidad de opiáceos después de la cesárea $(38,39)$.

$\mathrm{Si}$ bien en el presente estudio la prevalencia de complicaciones en la herida quirúrgica fue muy baja y dada las características del estudio, no se evaluó la asociación entre el uso de este fármaco con las alteraciones en la cicatrización de las heridas. Coincidentemente en otro estudio (38), la infiltración de un anestésico local produjo un sólo 
caso donde se complicó la herida quirúrgica con un hematoma, mientras que en esta serie se observó un caso de seroma. No obstante, otra investigación demostró que la administración continua de dosis usadas clínicamente de Bupivacaína en las heridas afectaba a la composición local de mediadores de la herida, siendo los cambios observados en la interleucina 10 compatibles con una alteración de los mecanismos antiinflamatorios, por tanto, esta modulación combinada con la liberación de los mediadores proinflamatorios como la sustancia $P$ requiere estudios futuros sobre la cicatrización de las heridas (40).

La presente investigación tiene un valor agregado por ser el primer estudio formal en realizarse en la institución ámbito de estudio en considerar el manejo del dolor posoperatorio y sus ventajas para la atención materna en garantizar una analgesia posoperatoria óptima que le permita a la puérpera tanto una recuperación y un apego con su recién nacido más temprano, como también recibir el alta hospitalaria más rápido, circunstancia que debe ser considerada y tomada en cuenta, sobre todo en instituciones de salud de carácter público, donde se observa con preocupación una atención del parto deshumanizada y donde no se ofrece ni se le garantiza a la usuaria el recibir una analgesia obstétrica ni en su posoperatorio, que le permitan vivir el nacimiento de sus hijos como una experiencia más placentera.

El estudio tiene limitaciones para la generalización de estos resultados, puesto que se tomó una muestra intencionada y si bien incluyó una muestra heterogénea, el tamaño muestral fue relativamente pequeño como para transpolar estos hallazgos al resto del universo de estudio. Asimismo, existieron ciertas limitaciones para el seguimiento de las pacientes y evaluar complicaciones tardías, pues al no existir una consulta posparto en la institución muchos de los casos evaluados se perdieron y debieron ser excluidas de la investigación.

\section{CONCLUSIÓN}

La infiltración de la incisión de Pfannenstiel con Bupivacaína al compararse con un placebo resultó ser efectiva para la analgesia posoperatoria en cesárea segmentaria, siendo un procedimiento simple y seguro que proporciona una reducción eficaz del dolor dentro de las primeras horas del puerperio inmediato, con un menor consumo de analgésicos, un tiempo mayor libre de dolor, con pocos y leves efectos colaterales. Recomendamos su utilización en las pacientes que son atendidas en esta insti- tución. Hacemos un llamado de alerta y reflexión a las autoridades del hospital como del Ministerio del Poder Popular para la Salud, para la creación de servicios de analgesia y terapia del dolor para el manejo del dolor agudo y crónico en todos los hospitales públicos venezolanos; de modo de que todas las usuarias que requieran atención materna puedan vivir una experiencia más agradable y humanizada del nacimiento, con una recuperación más rápida y un apego temprano a sus recién nacidos.

AGRADECIMIENTOS: Se agradece al personal médico del Servicio de Ginecología y Obstetricia del Hospital "Dr. Adolfo Pons" (IVSS) por su colaboración en la ejecución del protocolo de estudio.

\section{REFERENCIAS}

1. Faneite P, Rivera C, Faneite J, Amato R. Cesárea y mortalidad perinatal. Incidencia y tendencia 42 años (1969-2010). Rev Obstet Ginecol Venez 2012;72(3):145-51.

2. Uzcátegui $\mathrm{O}$, Cabrera $\mathrm{C}$. Aspectos bioéticos de la cesárea y consentimiento informado. Rev Obstet Ginecol Venez 2010;70(1):47-52.

3. Ceriani JM, Mariani G, Pardo A, Aguirre A, Pérez C, Brener $P$, et al. Nacimiuento por cesárea al término en embarazos de bajo riesgo: efectos sobre la morbilidad neonatal. Arch Argent Pediatr 2010;108(1):17-23.

4. Scott JR. Vaginal birth after cesarean delivery: a common-sense approach. Obstet Gynecol 2011;118(2 Pt 1):342-50.

5. Atkinson L, Drover DR, Clavijo CF, Carvalho B. Prior epidural lidocaine alters the pharmacokinetics and drug effects of extended-release epidural morphine (DepoDur) after cesarean delivery. Anesth Analg 2011;113(2):251-8.

6. Centers for Disease Control and Prevention. Tasas de parto por cesárea de 1970 a 2009. National Vital Statistics System [Base de datos en línea]. Disponible en: http://www.cdc.gov/nchs/nvss.htm. Acceso: 16 de Abril de 2014.

7. Solheim KN, Esakoff TF, Little SE, Cheng YW, Sparks $\mathrm{TN}$, Caughey AB. The effect of cesarean delivery rates on the future incidence of placenta previa, placenta accreta, and maternal mortality. J Matern Fetal Neonatal Med 2011;24(11):1341-6.

8. Faneite $\mathrm{P}$, Rivera C, Faneite J, Amato R. Cesárea y mortalidad perinatal. Incidencia y tendencia 42 años (1969-2010). Rev Obstet Ginecol Venez 2012;72(3):145-51.

9. American College of Obstetricians and Gynecologists. Vaginal birth after previous cesarean delivery. Practice Bulletin No. 115. Obstet Gynecol 2010; 116:45063.

10. Keïta-Meyer H. Postoperative analgesia after Caesarean section. Pain assessment and treatment. Editorial 
Elsevier: Amsterdam, The Netherlands. 2006. pp 67788.

11. Nguyen NK, Landais A, Barbaryan A, M'Barek MA, Benbaghdad $\mathrm{Y}, \mathrm{McGee} \mathrm{K}$, et al. Analgesic efficacy of Pfannenstiel incision infiltration with Ropivacaine 7.5 $\mathrm{mg} / \mathrm{mL}$ for caesarean section. Anesthesiol Res Pract 2010;2010. pii: 542375.

12. Pan PH, Coghill R, Houle TT, Seid MH, Lindel WM, Parker RL, et al. Multifactorial preoperative predictors for cesarean section pain and analgesic requirement. Anesthesiology 2006;104(3):417-25.

13. Gutiérrez W, Gutiérrez SE. Diferencias de sexo en el dolor. Una aproximación a la clínica. Rev Colomb Anestesiol 2012;40(3):207-12.

14. Agarwal K, Agarwal N, Agrawal VK, Agarwal A, Sharma M. Efficacy of single dose epidural morphine versus intermittent low-dose epidural morphine along with bupivacaine for postcaesarean section analgesia. Anesth Essays Res 2012;6(1):25-8.

15. Marcus HE, Fabian A, Dagtekin O, Schier R, Krep H, Böttiger BW, et al. Pain, postdural puncture headache, nausea and pruritus after cesarean delivery: a survey of prophylaxis and treatment. Minerva Anestesiol 2011;77(11):1043-9.

16. Harnett MJP, O'Rourke N, Walsh M, Carabuena JM, Segal S. Transdermal scopolamine for prevention of intrathecal morphine-induced nausea and vomiting after cesarean delivery. Anesth Analg 2007;105(3):7649.

17. Bergese SD, Ramamoorthy S, Patou G, Bramlett K, Gorfine SR, Candiotti KA. Efficacy profile of liposome bupivacaine, a novel formulation of bupivacaine for postsurgical analgesia. J Pain Res 2012;5:107-16.

18. Demiraran $Y$, Albayrak M, Yorulmaz IS, Ozdemir I. Tramadol and levobupivacaine wound infiltration at cesarean delivery for postoperative analgesia. $\mathrm{J}$ Anesth 2013;27(2):175-9.

19. Lavand'homme PM, Roelants F, Waterloos $\mathrm{H}$, De Kock MF. Postoperative analgesic effects of continuous wound infiltration with diclofenac after elective cesarean delivery. Anesthesiology 2007;6(6):1220-5.

20. Sakalli M, Ceyhan A, Uysal HY, Yazici I, Başar H. The efficacy of ilioinguinal and iliohypogastric nerve block for postoperative pain after caesarean section. Res Med Sci 2010;15(1):6-13.

21. Bamigboye AA, Justus HG. Local anaesthetic wound infiltration and abdominal nerves block during caesarean section for postoperative pain relief. Cochrane Database Syst Rev. 2009; 8 (3): CD006954.

22. Cusack SL, Reginald P, Hemsen L, Umerah E. The pharmacokinetics and safety of an intraoperative bupivacaine-collagen implant (XaraColl@) for postoperative analgesia in women following total abdominal hysterectomy. J Pain Res 2013;6(6):151-9.

23. Perniol A, Gupta A, Crafoord K, Darvish B, Magnuson $A$, Axelsson $K$. Intraabdominal local anaesthetics for postoperative pain relief following abdominal hysterectomy: a randomized, double-blind, dose-finding study. Eur Anaesthesiol 2009;26(5):421-9.

24. Rikalainen-Salmi R, Förster JG, Mäkelä K, Virolainen $P$, Leino KA, Pitkänen MT, et al. Local infiltration analgesia with levobupivacaine compared with intrathecal morphine in total hip arthroplasty patients. Acta Anaesthesiol Scand 2012;56(6):695-705.

25. Ozyilmaz K, Ayoglu H, Okyay RD, Yurtlu S, Koksal $\mathrm{B}$, Hanci V, et al. Postoperative analgesic effects of wound infiltration with tramadol and levobupivacaine in lumbar disk surgeries. J Neurosurg Anesthesiol 2012;24(4):331-5.

26. Bilgin TE, Bozlu M, Atici S, Cayan S, Tasdelen B. Wound infiltration with bupivacaine and intramuscular diclofenac reduces postoperative tramadol consumption in patients undergoing radical retropubic prostatectomy: a prospective, double-blind, placebo-controlled, randomized study. Urology 2011;78(6):1281-5.

27. Memis D, Hekimoglu S, Kaya G, Atakan HI, Kaplan M. Efficacy of levobupivacaine wound infiltration with and without intravenous lornoxicam for post-varicocoele analgesia: a randomized, double-blind study. Clin Drug Investig 2008;28 (6):353-9.

28. Kaki AM, Al Marakbi W. Post-herniorrhapy infiltration of tramadol versus bupivacaine for postoperative pain relief: a randomized study. Ann Saudi Med 2008;28(3):165-8.

29. Nesioonpour S, Akhondzadeh R, Pipelzadeh MR, Rezaee S, Nazaree E, Soleymani M. The effect of preemptive analgesia with bupivacaine on postoperative pain of inguinal hernia repair under spinal anesthesia: a randomized clinical trial. Hernia 2013;17(4):465-70.

30. Jha AK, Bhardwaj N, Yaddanapudi S, Sharma RK, Mahajan JK. A randomized study of surgical site infiltration with bupivacaine or ketamine for pain relief in children following cleft palate repair. Paediatr Anaesth 2013;23(5):401-6.

31. Ergil J, Akkaya T, Gozaydin O, Gunsoy B, Alicura $\mathrm{S}$, Aladag $\mathrm{E}$, et al. Vasoconstrictive and analgesic efficacy of locally infiltrated levobupivacaine in tonsillectomy patients. Int J Pediatr Otorhinolaryngol 2012;76(10):1429-33.

32. Gupta A. Wound infiltration with local anaesthetics in ambulatory surgery. Curr Opin Anaesthesiol 2010;23(6):708-13.

33. Bamigboye AA, Justus HG. Caesarean section wound infiltration with local anaesthesia for postoperative pain relief - any benefit? S Afr Med J 2010;4(5):313-9.

34. Lluna E. Infusión continúa de anestésico local a nivel subfascial como analgesia postoperatoria en la cesárea. Evaluación clínica y de satisfacción. Doctorado en Medicina y Cirugía. Universitat Autónoma de Barcelona. 2010. [Tesis en línea]. Disponible en: http:// ddd.uab.es/record/90446? In=es. Acceso: 22 de Mayo de 2014.

35. Jabalameli M, Safavi M, Honarmand A, Saryazdi H, Moradi D, Kashefi P. The comparison of intraincisional injection tramadol, pethidine and bupivacaine on postcesarean section pain relief under spinal anesthesia. Adv Biomed Res 2012;1:53

36. Momani O. Controlled trial of wound infiltration with bupivacaine for post operative pain relief after caesarean section. Bahrain Med Bull 2001;23(2):83-5.

37. Ducarme G, Sillou S, Wernet A, Davitian C, Poujade $O$, Ceccaldi PF, et al. Single-shot ropivacaine wound 
infiltration during cesarean section for postoperative pain relief. Gynecol Obstet Fertil 2012;40(1):10-3.

38. Bensghir M, Elwali A, Miller C, Azendour H, Drissi M, Bakkali $\mathrm{H}$, et al. Effects of skin infiltration with ropivacaine $0.75 \%$ on postoperative pain after caesarean section. Gynecol Obstet Fertil 2008;36(5):516-20.

39. Bamigboye AA, Justus HG. Ropivacaine abdominal wound infiltration and peritoneal spraying at cesarean delivery for preemptive analgesia. J Gynaecol Obstet 2008;102(2):160-4.

40. Carvalho B, Clark DJ, Yeomans DC, Angst MS. Continuous subcutaneous instillation of bupivacaine compared to saline reduces Interleukin 10 and increases Substance $P$ in surgical wounds after cesarean delivery. Anesth Analg 2010;111(6):1452-9. 